\title{
Effect of Vitamin C on Serum Concentration of Brain-Derived Neurotrophic Factor among Healthy Inactive Young Men
}

\section{Hossein Nazari' ${ }^{1}$, Sajad Heydarpoor ${ }^{2}$, Abuzar Mohamadi Mofrad ${ }^{3 *}$, Mazgaldi Nazari $^{3}$, Araz Nazari ${ }^{4}$ \\ ${ }^{1}$ Faculty of Physical Education and Sport Sciences, University of Mazandaran, Mazandaran, Iran}

${ }^{2}$ Faculty of Physical Education and Sport Sciences, Science and Research Kohgiloyeh and Boyerahmad Branch, Islamic Azad University, Kohgiloyeh and Boyerahmad, Iran

${ }^{3}$ Department of Sport Physiology, Shahid Chamran University of Ahvaz, Ahvaz, Iran

${ }^{4}$ Higher Educational Complex of Saravan, Saravan, Iran

\section{A BSTRACT}

Introduction: The aim of this study was to investigate the effect of consuming vitamin $\mathrm{C}$ on the serum level of Brain-derived neurotrophic factor (BDNF) among healthy inactive young men. Materials and Methods: In this semi-empirical study, 18 healthy inactive men were randomly divided into two groups; a group consuming vitamin $\mathrm{C}$ as a supplement and a control group. Subjects in consuming vitamin $\mathrm{C}$ group received 500 milligrams of vitamin $\mathrm{C}$ supplement daily for one week. The control group consumed placebo. Blood samples of participants were taken in fasting mode to measure BDNF concentration. BDNF was measured by ELISA method. Results: The serum levels of BDNF significantly increased one week after receiving vitamin $\mathrm{C}$ compared to the control group. In addition, the level of BDNF in subjects received vitamin $\mathrm{C}$ for one week significantly increased compared to the beginning of the investigation. Conclusion: Data indicate that consuming vitamin $\mathrm{C}$ increased the serum concentration of BDNF in healthy inactive men.

\section{Key words:}

1. Brain-Derived Neurotrophic Factor

2. Ascorbic Acid

3. Men

* Corresponding Author: Abuzar Mohamadi Mofrad

E-mail: Abuzar.mofrad@gmail.com 


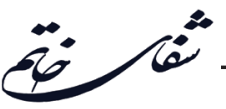

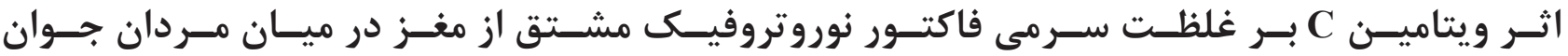

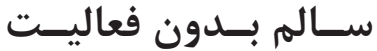

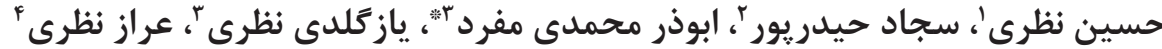 \\ 'دانشكده تربيت بدنى و علوم ورزشى، دانشخاه مازندران، مازندران، ايران

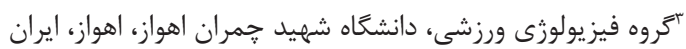

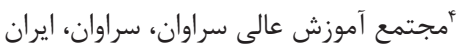

'دانشكده تربيت بدنى و علوم ورزشى، واحد علوم و تحقيقات كهگيلويه و بويراحمد، دانشخاه آزاد اسلامى، كهَيلويه و بويراحمد، ايران

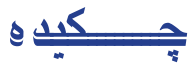

مقدمه: هدف از اين مطالعه بررسى اثر مصرف ويتامين C بر سطح سرمى فاكتور نوروتروفيك مشتق از

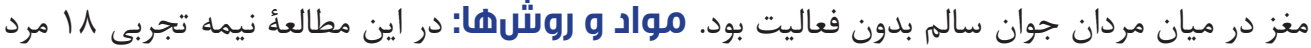

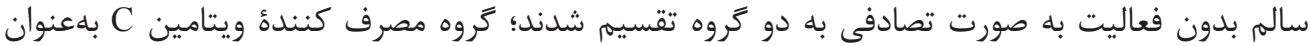

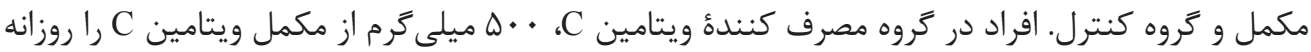

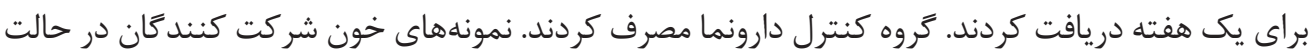

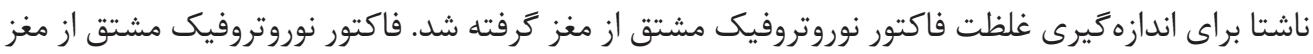

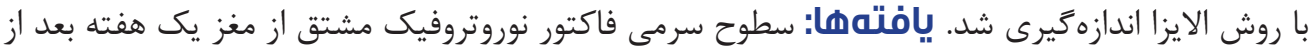

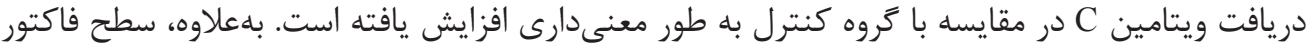

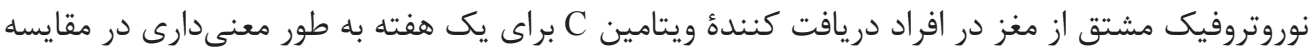

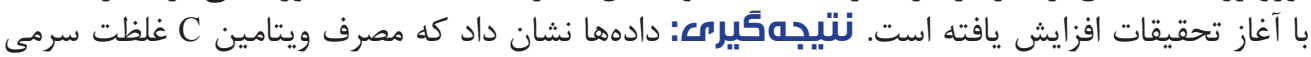

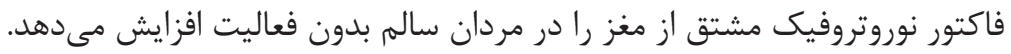

كليد وازهها:

ا إ. فاكتور نوروتروفيك مشتق

از مغن

r.

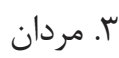

* نويسنده مسئول: ابوذر محمدى مفرد آدرس الكترونيكى: Abuzar.mofrad@gmail.com 


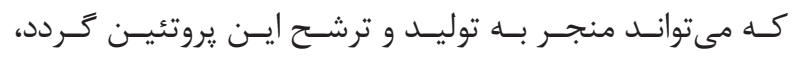

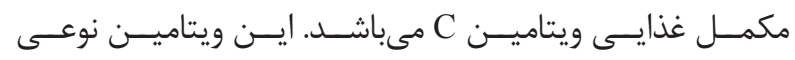

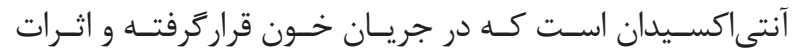

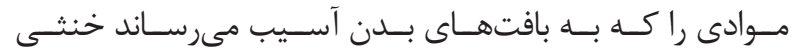

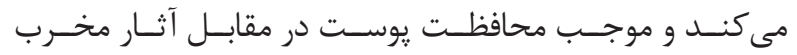

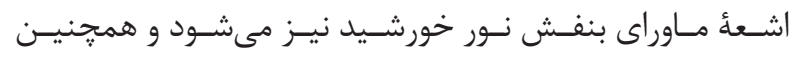

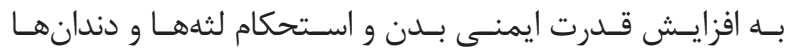

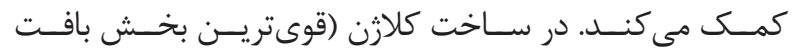

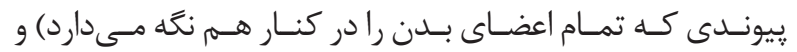

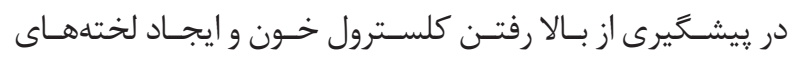

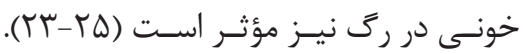

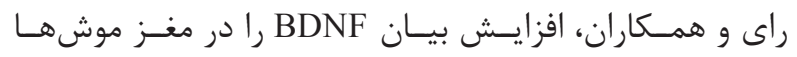

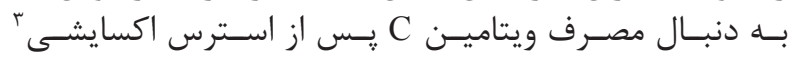

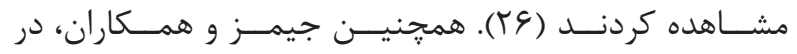

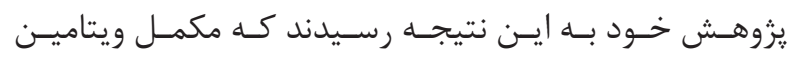

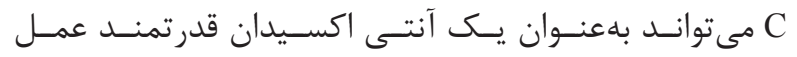

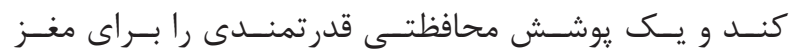

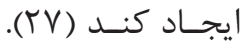

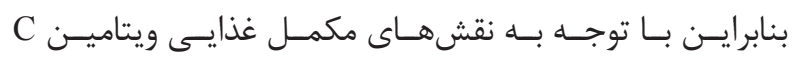

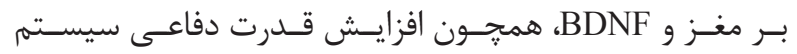

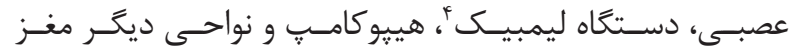

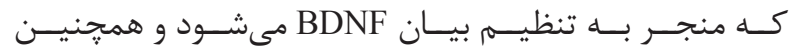

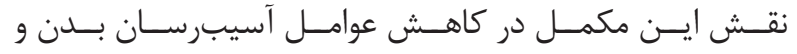

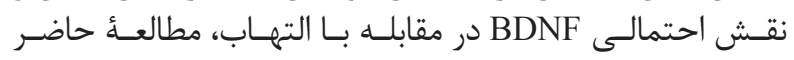

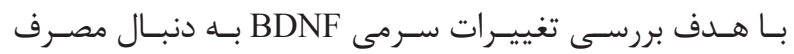

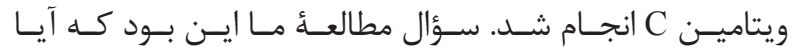

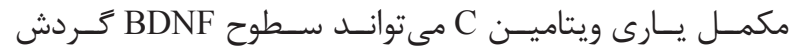

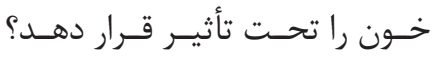

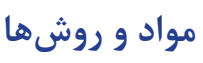

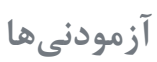

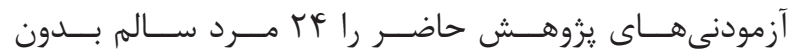

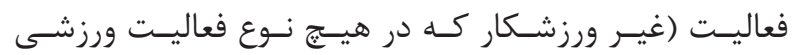

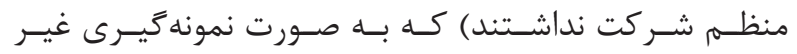

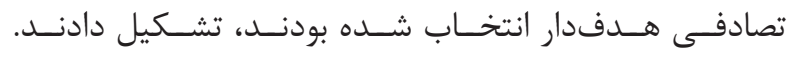

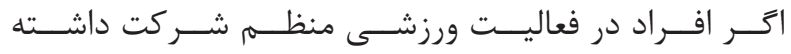

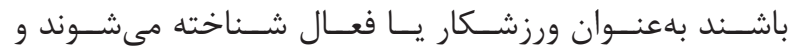

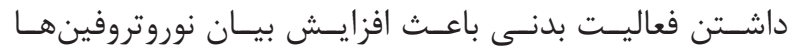

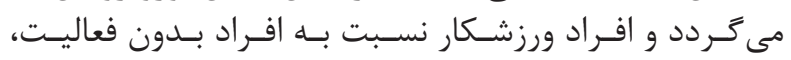

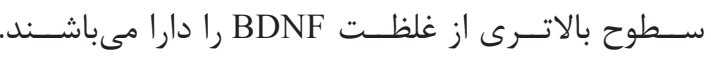

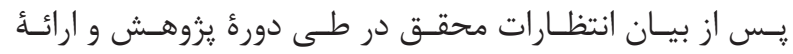

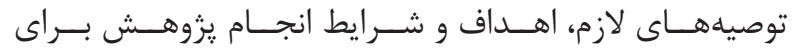

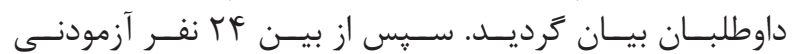

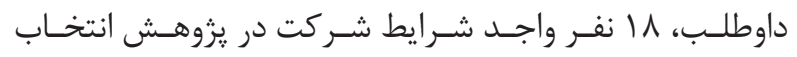

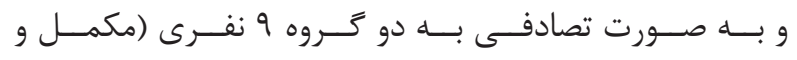

${ }^{1}$ Neurotrophins

${ }^{2}$ Brain-derived neurotrophic factor

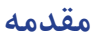

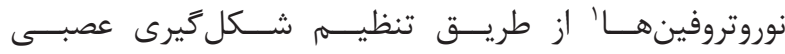

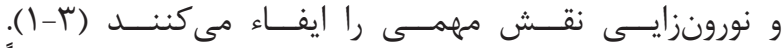

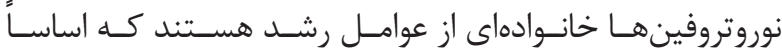

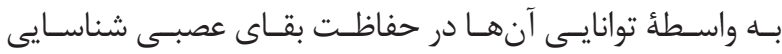

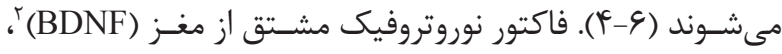

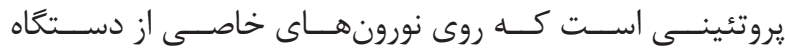

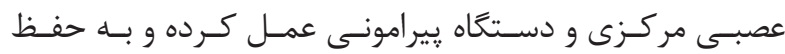

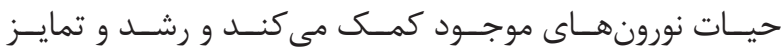

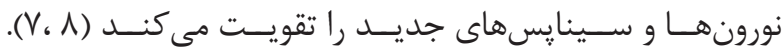

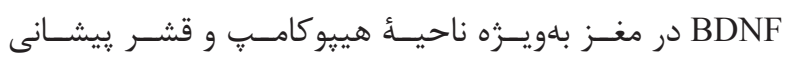

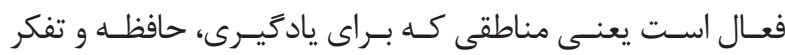

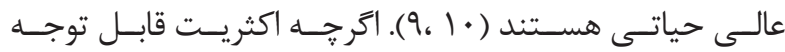

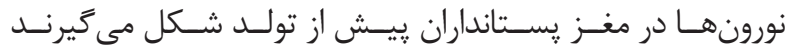

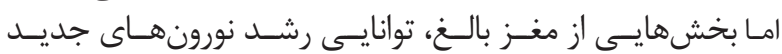

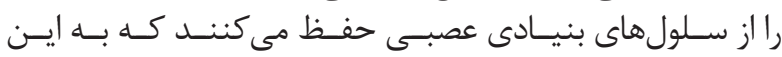

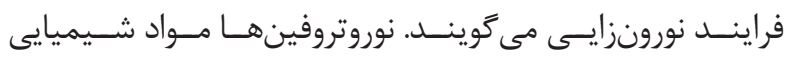

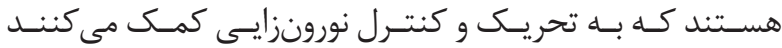

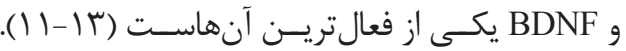

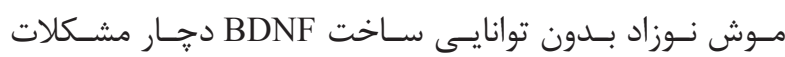

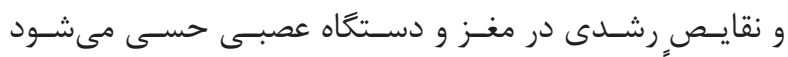

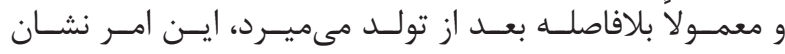

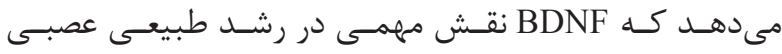

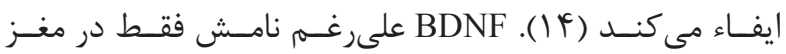

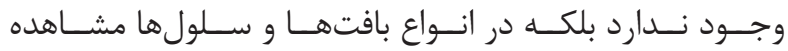

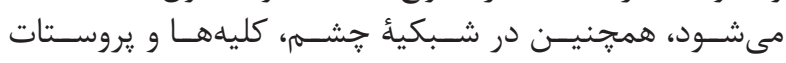

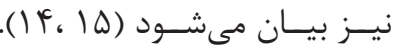

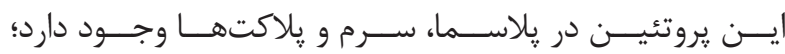

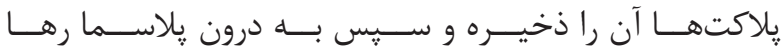

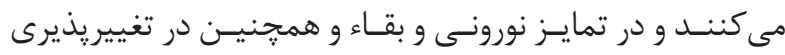

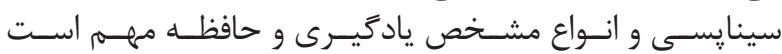

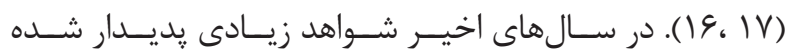

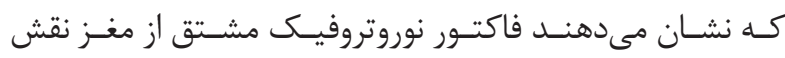

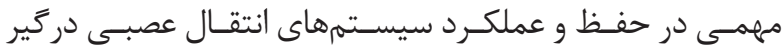

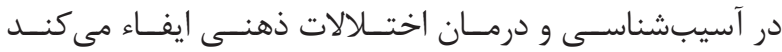

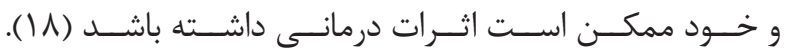

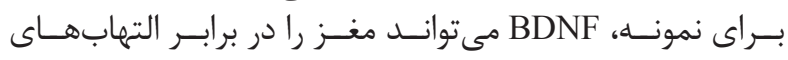

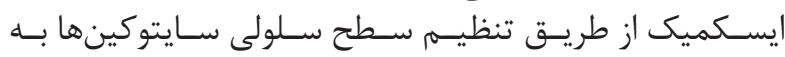

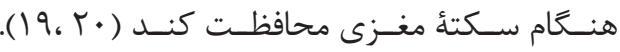

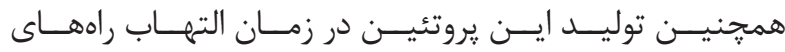

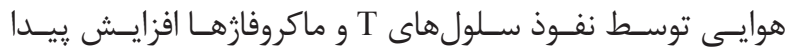

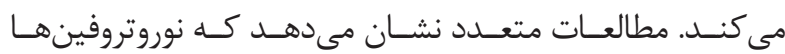

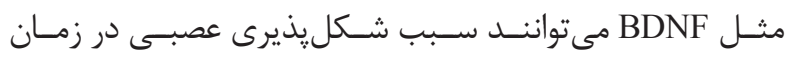

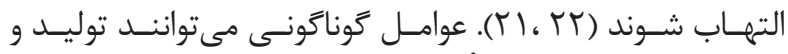

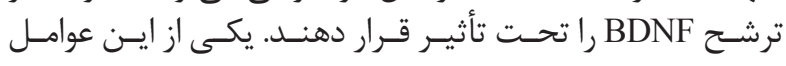

${ }^{3}$ Oxidative stress

${ }^{4}$ Limbic system 


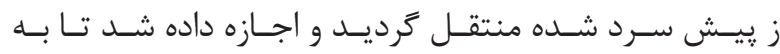

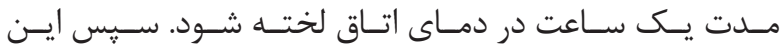

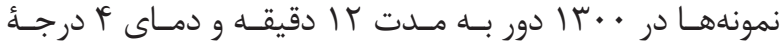

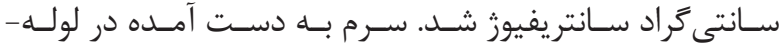

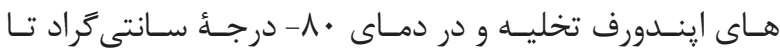

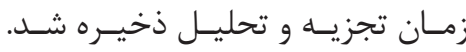

اندازهيرى BDNF

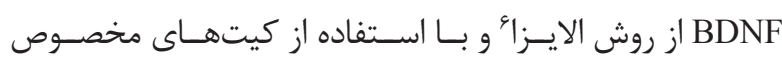

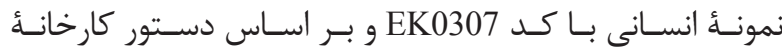

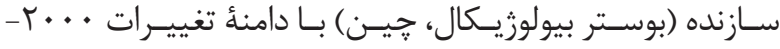

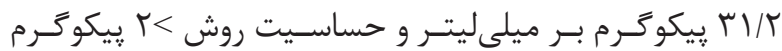

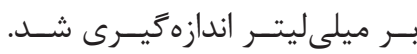

تجزيه و تحليل دادهها

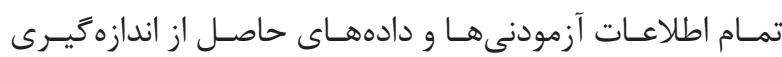

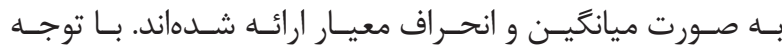

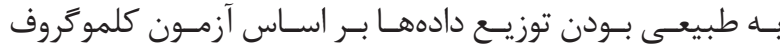

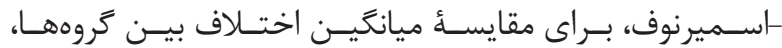

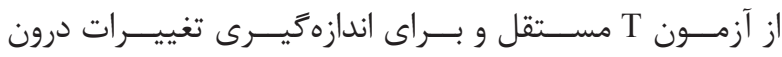

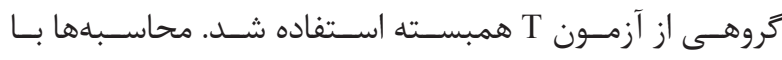

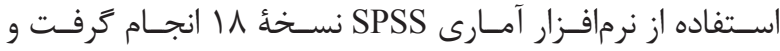

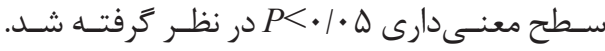

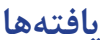

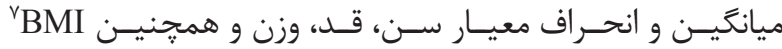

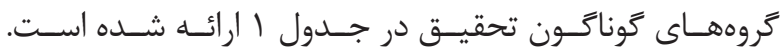

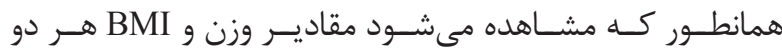

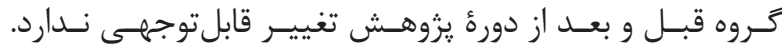

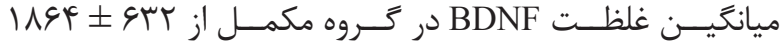

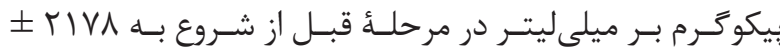

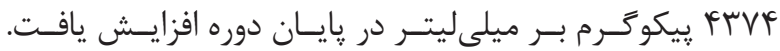

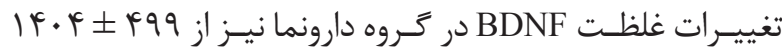

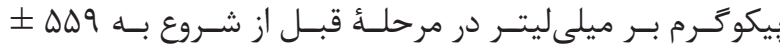

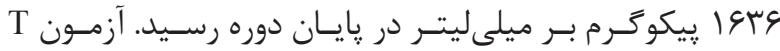

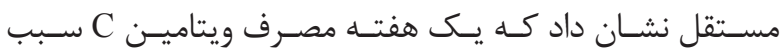

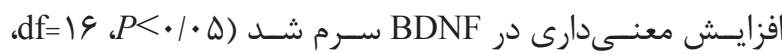

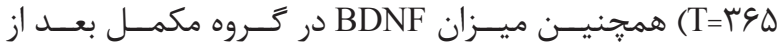

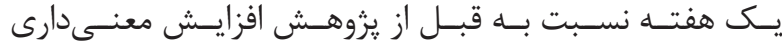

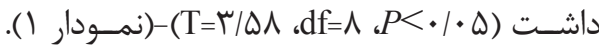

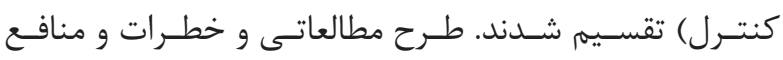

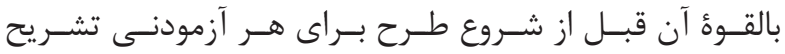

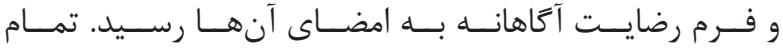

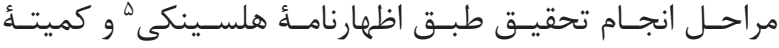

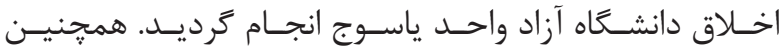

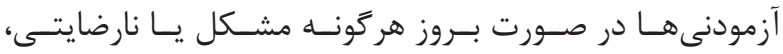

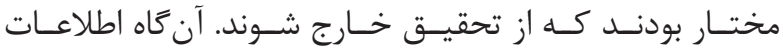

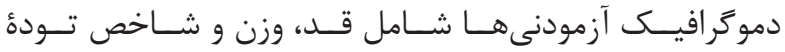

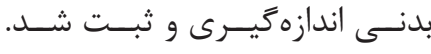
شر ايط ورود و خروج از مطالعه

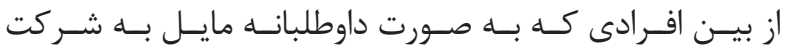

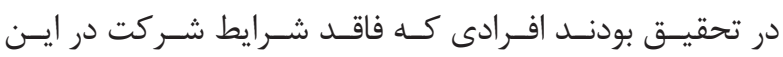

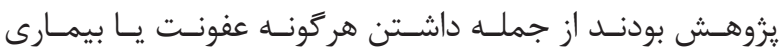

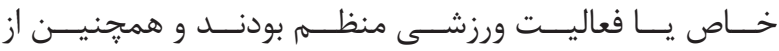

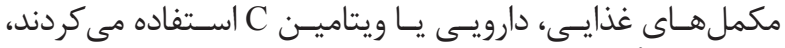

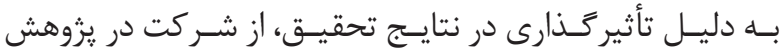

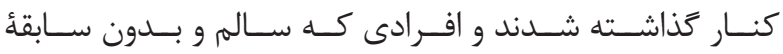

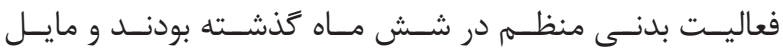

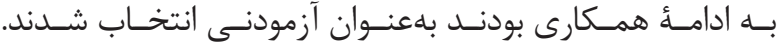

$$
\text { تهيئ مكمل و ميزان مصرف }
$$

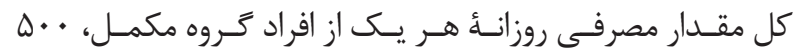

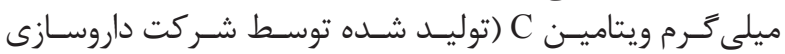

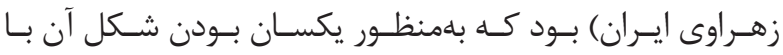

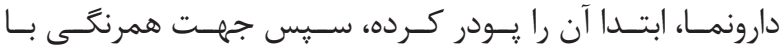

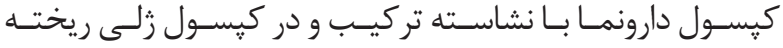

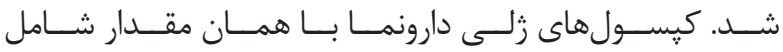

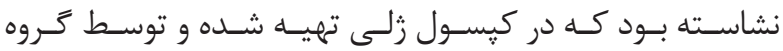

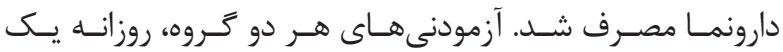

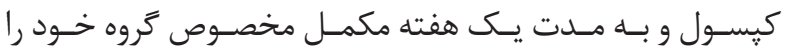

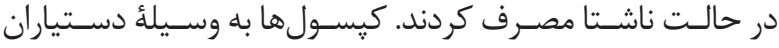

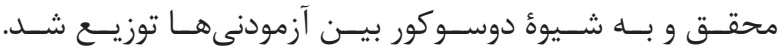

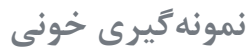

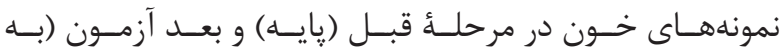

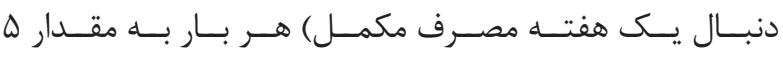

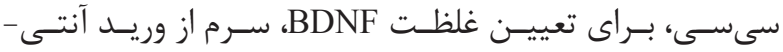

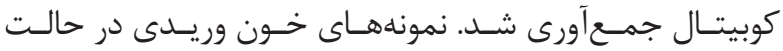

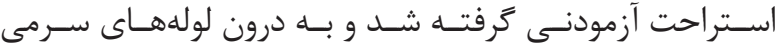

\begin{tabular}{|c|c|c|c|c|c|c|}
\hline \multicolumn{2}{|c|}{ BMI (كيلوكرم تقسيم بر مجذور قد) } & \multicolumn{2}{|c|}{ وزن (كيلوكرم) } & \multirow{2}{*}{ قد (سانتىمتر) } & \multirow{2}{*}{ سن (سال) } & \multirow{2}{*}{ 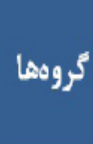 } \\
\hline بعد & 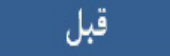 & بعد & 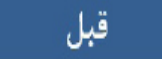 & & & \\
\hline$r M / F Y \pm T / V V$ & $r / r q \pm r / .4$ & $G H / F Y \pm N / Y Y$ & $G H / N D \pm 9 / r$. & $|V r /| F \pm r / \Lambda q$ & $r r / I F \pm 1 / r F$ & عكمل \\
\hline$r r / q_{0} \pm 1 / 9$. & $r Y / F Y \pm I / Y \Lambda$ & $V I / V I \pm F / Q T$ & $V / R T \pm r / Q$ & $I V N / \Delta V \pm V / I I$ & $r r / \Lambda Q \pm r / Q F$ & 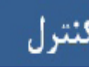 \\
\hline
\end{tabular}

جدول ا- ميانكَين و انحراف استاندارد ويثَى هاى آزمودنىهاى كروههاى مختلف قبل و بعد از يروهش.

${ }^{5}$ Helsink

${ }^{6}$ Elisa

${ }^{7}$ Body mass index 


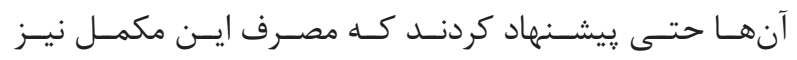

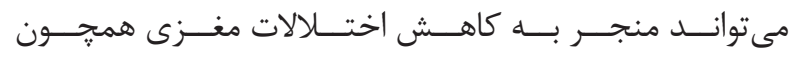

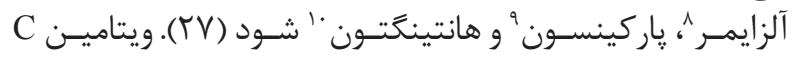

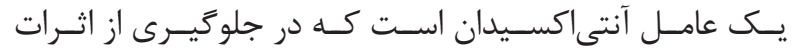

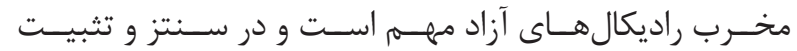

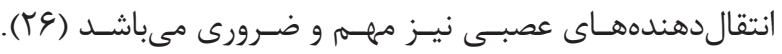

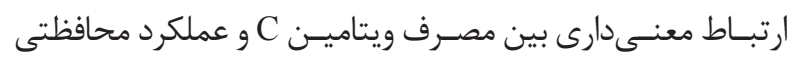

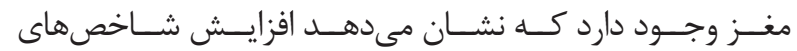

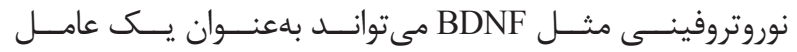

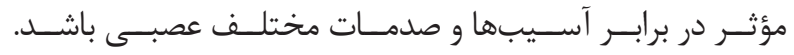

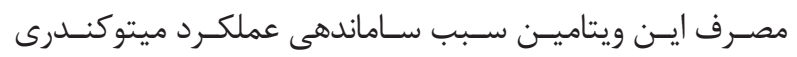

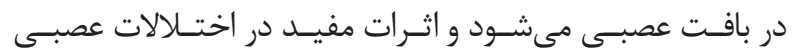

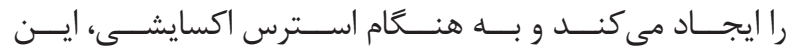

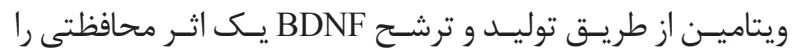

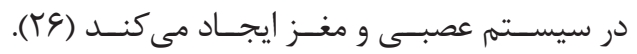

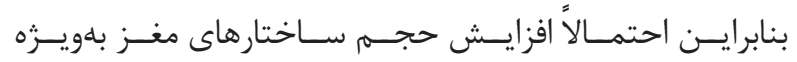

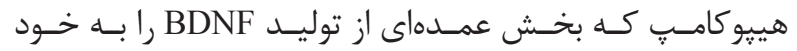

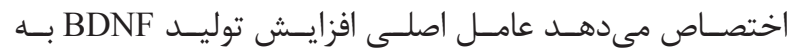

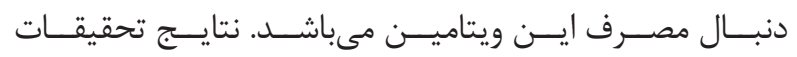

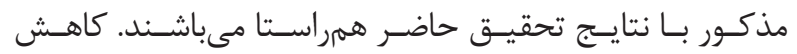

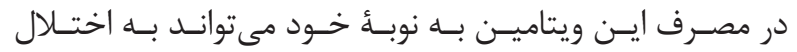

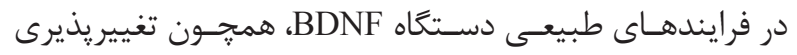

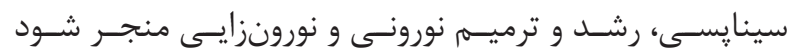

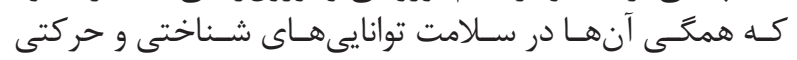

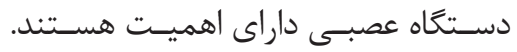

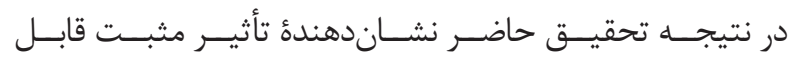

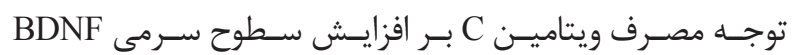

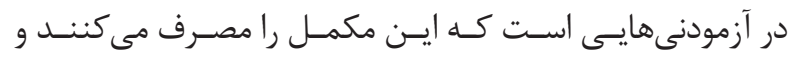

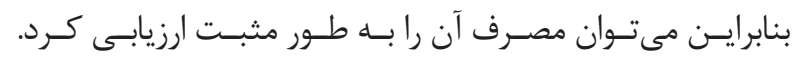

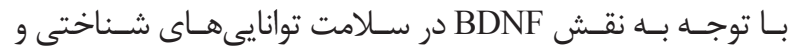

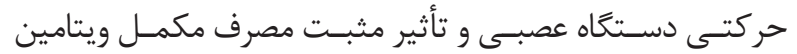

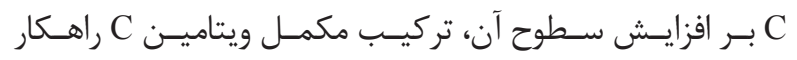

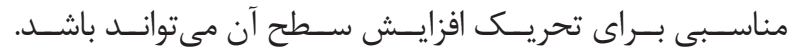

1. Goekint M, De Pauw K, Roelands B, Njemini R, Bautmans I, Mets T, et al. Strength training does not influence serum brain-derived neurotrophic factor. Eur J Appl Physiol. 2010; 110(2): 285-93.

2. Patapoutian A, Reichardt LF. Trk receptors: mediators of neurotrophin action. Curr.Opin.Neurobiol. 2001; 11(3): 272-80.

3. Miller FD, Kaplan DR. Neurotrophin signalling

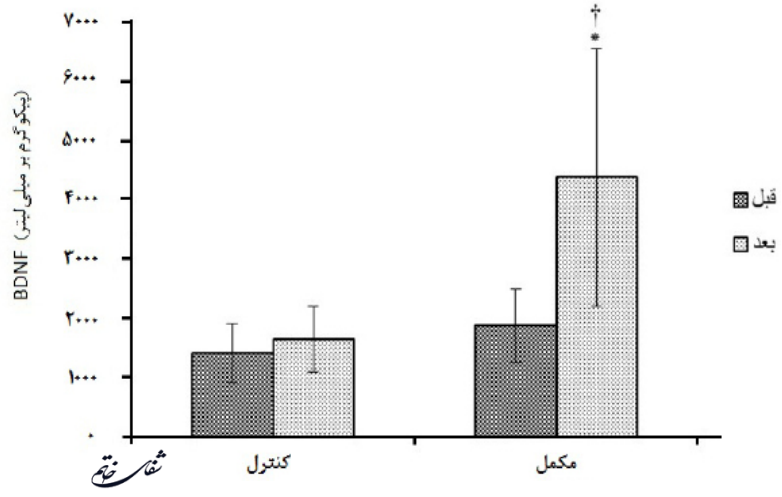

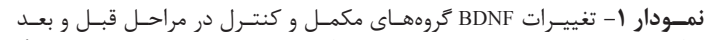

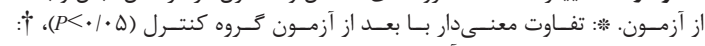

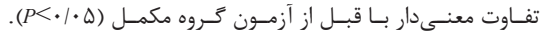

\section{بحث و نتيجه كيرى}

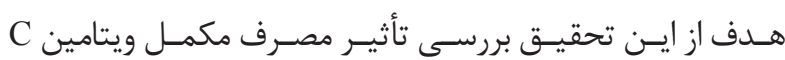

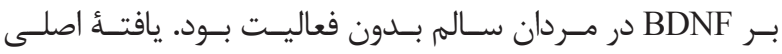

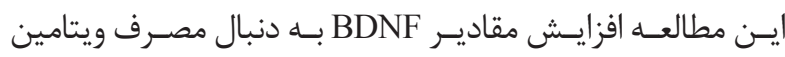

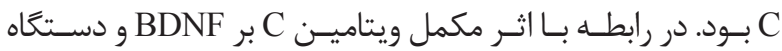

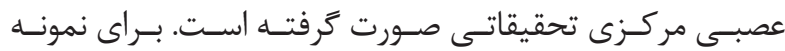

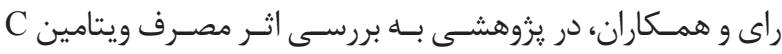

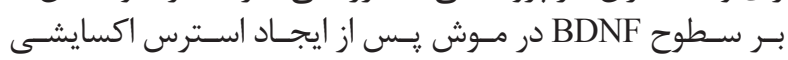

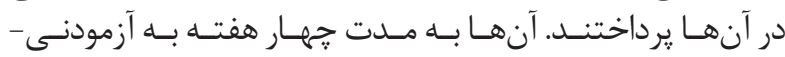

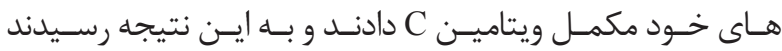

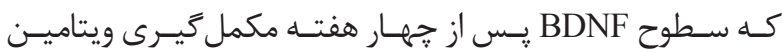

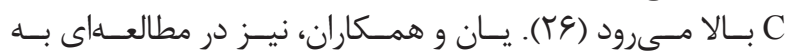

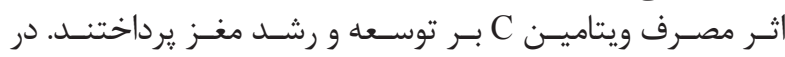

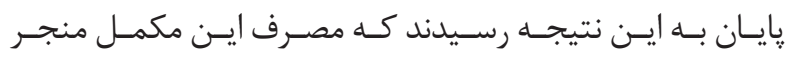

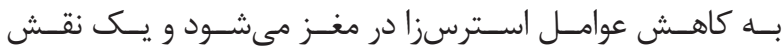

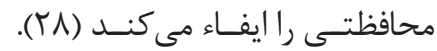

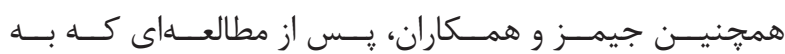

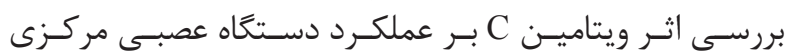

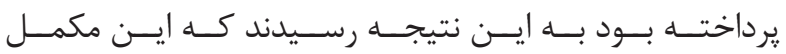

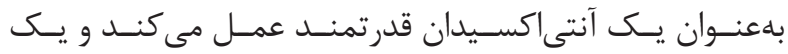

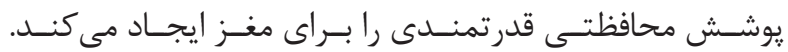

منابع

pathways regulating neuronal apoptosis. Cell Mol.Life Sci. 2001; 58(8): 1045-53.

4. Segal E, Shapira M, Regev A, Peer D, Botstein D, Koller D, et al. Module networks: identifying regulatory modules and their condition-specific regulators from gene expression data. Nat.Genet. 2003; 34(2): 166-76.

5. Huang EJ, Reichardt LF. Trk receptors: roles in neuronal signal transduction. Annu.Rev.Biochem.

\footnotetext{
${ }^{8}$ Alzaymer

${ }^{9}$ Parkinson

${ }^{10}$ Huntington
} 


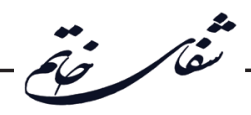

$2003 ; 72: 609-42$.

6. Miller FD, Kaplan DR. Signaling mechanisms underlying dendrite formation. Curr.Opin.Neurobiol. 2003; 13(3): 391-8.

7. Acheson A. Conover JC, Fandl JP, DeChiara TM, Russell M, Thadani A, et al. A BDNF autocrine loop in adult sensory neurons prevents cell death. Nature. 1995; 374(6521): 450-3.

8. Huang EJ, Reichardt LF. Neurotrophins: roles in neuronal development and function. Annu Rev Neurosci. 2001; 24: 677-736.

9. Yamada K, Nabeshima T. Brain-derived neurotrophic factor/TrkB signaling in memory processes. J. Pharmacol. Sci. 2003; 91(4): 267-70.

10. Bekinschtein P, Cammarota M, Katche C, Slipczuk L, Rossato JI, Goldin A, et al. BDNF is essential to promote persistence of long-term memory storage. Proc Natl Acad Sci. U.S.A. 2008; 105(7): 2711-6.

11. Benraiss A, Chmielnicki E, Lerner K, Roh D, Goldman SA. Adenoviral brain derived neurotrophic factor induces both neostriatal and olfactory neuronal recruitment from endogenous progenitor cells in the adult forebrain. J Neurosci. 2001; 21(17): 6718-31.

12. Pencea V, Bingaman KD, Wiegand SJ, Luskin MB. Infusion of brain-derived neurotrophic factor into the lateral ventricle of the adult rat leads to new neurons in the parenchyma of the striatum, septum, thalamus, and hypothalamus. J Neurosci. 2001; 21(17): 6706-17.

13. Zigova T, Pencea V, Wiegand SJ, Luskin MB. Intraventricular administration of BDNF increases the number of newly generated neurons in the adult olfactory bulb. Mol Cell Neurosci. 1998; 11(4): 234-45.

14. Ernfors P, Kucera J, Lee KF, Loring J, Jaenisch R. Studies on the physiological role of brain-derived neurotrophic factor and neurotrophin-3 in knockout mice. Int J Dev Biol. 1995; 39(5): 799-807.

15. Tanaka T, Saito H, Matsuki N. Inhibition of GABAA synaptic responses by brain derived neurotrophic factor (BDNF) in rat hippocampus. J Neurosci. 1997; 17(9): 2959-66.

16. Lommatzsch M, Zingler D, Schuhbaeck K, Schloetcke K, Zingler C, Schuff-Werner P, et al. The impact of age, weight and gender on BDNF levels in human platelets and plasma. Neurobiol Aging. 2005; 26(1): 115-23.

17. Ploughman M, Granter-Button S, Cherneko G, Tucker BA, Mearow KM, Corbett D. Endurance exercise regimens induce differential effects on brain-derived neurotrophic factor, synapsin-I and insulin-like growth factor I after focal ischemia. Neuroscience. 2005; 136(4): 991-1001.

18. Russo-Neustadt A, Ha T, Ramirez R, Kesslak JP. Physical activity antidepressant treatment combination: impact on brain-derived neurotrophic factor and behavior in an animal model. Behav Brain Res. 2001; 120(1): 87-95.

19. Jiang Y, Wei N, Lu T, Zhu J, Xu G, Liu X. Intranasal brain-derived neurotrophic factor protects brain from ischemic insult via modulating local inflammation in rats. Neuroscience. 2011; 172: 398-405.

20. Makar TK, Trisler D, Sura KT, Sultana S, Patel N, Bever CT. Brain derived neurotrophic factor treatment reduces inflammation and apoptosis in experimental allergic encephalomyelitis. J Neurol Sci. 2008; 270(1-2): 70-6.

21. Braun A, Lommatzsch M, Neuhaus-Steinmetz U, Quarcoo D, Glaab T, McGregor GP, et al. Brain-derived neurotrophic factor (BDNF) contributes to neuronal dysfunction in a model of allergic airway inflammation. Br J Pharmacol. 2004; 141(3): 431-40.

22. Watanabe M, Endo Y, Kimoto K, Katoh-Semba R, Arakawa Y. Functional regulation of tactile sense by brain-derived neurotrophic factor in adult rats during acute inflammation. Neuroscience. 2000; 97(1): 171-5.

23. El-Sokkary GH. Awadalla EA. The protective role of vitamin $\mathrm{C}$ against cerebral and pulmonary damage induced by cadmium chloride in male adult albino rat. Open Endocrinol J. 2011; 4: 1-8.

24. Podmore ID, Griffiths HR, Herbert KE, Mistry N, Mistry $\mathrm{P}$, Lunec J. Vitamin C exhibits pro-oxidant properties. Nature. 1998; 392(6676): 559.

25. Coskun S, Gonul B, Guzel NA, Balabanli B. The effects of vitamin $\mathrm{C}$ supplementation on oxidative stress and antioxidant content in the brains of chronically exercised rats. Mol Cell Biochem. 2005; 280(1-2): 135-8.

26. Rai A, Madhyastha S, Rao G, Rai R, Sahu S. A comparison of resveratrol and vitamin $\mathrm{C}$ therapy on expression of BDNF in stressed rat brain homogenate. IOSRJ Pharm. 2013; 3(10): 22-7.

27. James M, May MD. Vitamin C transport and its role in the central nervous system. Subcell Biochem. 2012; 56: 85-103.

28. Yan J, Studer L, McKay R. Ascorbic acid increases the yield of dopaminergic neurons derived from basic fibroblast growth factor expanded mesencephalic precursors. J Neurochem. 2001; 76(1): 307-11. 